Annuaire suisse de politique de développement

2 | 1982

Annuaire Suisse - Tiers Monde 1982

\title{
Agir sur les causes du mal développement
}

Gegen die Ursachen der Fehlentwicklung Handeln

Mario Carera

\section{OpenEdition}

\section{Journals}

Édition électronique

URL : http://journals.openedition.org/aspd/1127

ISSN : 1663-9669

Éditeur

Institut de hautes études internationales et du développement

Édition imprimée

Date de publication : 31 août 1982

Pagination : 155-159

ISSN : 1660-5934

\section{Référence électronique}

Mario Carera, "Agir sur les causes du mal développement », Annuaire suisse de politique de développement [En ligne], 2 | 1982, mis en ligne le 23 janvier 2013, consulté le 07 septembre 2020. URL : http://journals.openedition.org/aspd/1127 


\title{
AGIR SUR LES CAUSES DU MAL DEVELOPPEMENT
}

\author{
Mario Carera
}

Secrétaire de la section romande de la Déclaration de Berne

\section{GEGEN DIE URSACHEN DER FEHLENTWICKLUNG HANDELN}

Zusammenfassung : Die Erklärung von Bern wurde 1968 in der Form eines Manifestes gegründet, das vom Bundesrat die Erhöhung der öffentlichen Mittel für Entwicklungszusammenarbeit, die Verminderung der militärischen Ausgaben und einen gerechten Preis für Rohstoffe forderte. 1970 wurde die EvB ein Verein. Regiionale Stellen wurden eröffnet und ermöglichten dadurch eine bessere und kontinuierliche Informationsarbeit. 1973 leistete die EvB eine grosse Manifestation gegen den portugiesischen Kolonialismus. Die EvB verbreitet Information über Grundprodukte, wie z.B. Kaffee und Jute. 1975 erschien der Bericht über "Entwicklungsland Welt - Entwicklungsland Schweiz", der zum Referenztext der EvB wurde. Die Welt wird nicht mehr in Nord-Süd geteilt, sondern in Zentrum und Peripherie. Die EvB gibt auch Berichte über die Tätigkeit der schweizerischen Multinationalen in der Dritten Welt heraus. Die EvB war auch am "Symposium der Solidarität" 1978 in der Westschweiz und 1981 in Bern auf nationaler Ebene beteiligt. Gruppen werden gebildet, die Themen wie das Bankgeheimnis, die Kinderliteratur oder Tourismus und Entwicklung analysieren.

La Déclaration de Berne (DB) est née en 1968 sous la forme d'un Manifeste exigeant du Gouvernement suisse, entre autres choses, I'accroissement de I'aide publique au développement, la diminution des dépenses militaires à cette fin, le paiement des matières premières à un juste prix, l'ouverture des programmes scolaires à la problématique du développement... Le document portait les signatures de plus de dix mille personnes qui prenaient simultanément l'engagement de consacrer un pourcentage de leur revenu à des œuvres d'entraide. Le Manifeste regroupait principalement des personnes issues de milieux ecclésiastiques et des citoyens mobilisés par le sort du Tiers Monde. L'objectif général était de sensibiliser le peuple suisse à la misère et à la faim croissantes. Dépassant la vision missionnaire et caritative traditionnelle, le Manifeste déplorait les inégalités entre pays riches et pays pauvres, tout en appelant à la constitution d'une "volonté nationale" capable d'infléchir les autorités fédérales vers une politique plus généreuse à 
l'égard des pays de l'hémisphère Sud.

Le premier résultat de ce Manifeste - en plus de son effet public - fut un accroissement des fonds dont purent disposer les organisations traditionnelles d'aide.

\section{Une action continue}

Les initiateurs du Manifeste s'aperçurent très rapidement de la nécessité de mener une action continue et d'aborder, de manière permanente, les aspects politiques du développement. En 1970, la DB se constitue en Association reconnaissant par là-même la nécessité d'un travail prolongé d'information et de sensibilisation politique. Des secrétariats régionaux sont créés et financés par des membres et sympathisants qui croient plus que d'autres à l'urgence d'une évolution dans la mentalité et les structures des pays industrialisés (la Suisse en l'occurrence). Le Manifeste de la DB est complété par un nouveau document où certaines causes politiques - au Nord - du sous-développement du Sud commencent à être explicitées : termes de l'échange, centre de décision à l'étranger, accueil secret de capitaux fuyant leurs obligations fiscales, etc. II est reconnu aussi que ces diverses réalités publiques ou clandestines constituent une forme de violence qui provoque dans le Tiers Monde une autre violence, révolutionnaire celle-là.

\section{Les premières campagnes}

A l'occasion de la présence du Portugal au Comptoir suisse de Lausanne en 1973 , la DB élève sa première grande protestation publique et participe à un large mouvement de sensibilisation sur le colonialisme portugais. Elle est aussi partie prenante à l'initiative en vue d'interdire les exportations d'armes (proposition refusée de justesse en vote populaire). Un journal bimestriel est lancé : "Vers un développement SOLIDAIRE".

En 1974 et 1976-77 sont entreprises de grandes campagnes nationales d'information ayant chacune pour support un produit de base importé du Tiers Monde : L'action "Ujamaa" (café de Tanzanie) et l'action "jute" (sacs de jute du Bangladesh). Les conditions de vie dans les pays producteurs, le marché des matières premières et, pour l'action jute, les phénomènes écologiques - le rapport avec la concurrence jute/plastique - constituent les axes principaux de ces deux campagnes. C'est dans la mouvance de ces actions que se créent, dès 1974-75, les Magasins du Monde lobjectif : informations sur le Tiers Monde par la vente de ses produits).

En 1976, à l'occasion de la consultation populaire relative à un crédit de la Confédération à I'IDA (Association Internationale pour le développement), la DB met en cause le modèle de développement préconisé et encouragé par la Banque mondiale. A la même époque, est discutée et adoptée au Parlement la loi sur la coopération au développement, pas positif vers une Suisse plus ouverte aux nécessités d'une coopération au service des pauvres et opprimés du Tiers Monde. 


\section{Une nouvelle analyse}

Sur la scène des nations, cette période est marquée par le climat de crise économique et sociale, crise attribuée d'abord bien légèrement à la "crise du pétrole". L'idée d'un nouvel ordre économique international, mal défini, tend à devenir la panacée dans tous les forums onusiens... Multinationales et Etats - du Nord comme du Sud - tissent des réseaux de pouvoir de plus en plus serrés, réseaux dont de larges couches de la population sont bien souvent absentes. C'est à la même époque (1975) que paraît le rapport "Maldéveloppement Suisse/Monde", fruit d'une large réflexion commune des différentes organisations de développement. Ce rapport est discuté et adopté par la DB comme texte de référence. Cette analyse ne divise plus le monde en Nord et Sud, mais en Centre et Périphérie, définissant par là de nouvelles solidarités entre les "défavorisés" à l'intérieur des nations, au Nord comme au Sud.

Le développement ne se mesure plus en terme de croissance du produit national brut, mais en terme de libération économique, politique, sociale et culturelle.

\section{Des actions fort diverses}

Cette nouvelle orientation engage aussi la DB dans des actions plus politiques. Citons par exemple :

- La publication de cas exemplaires, impliquant des entreprises suisses dans des agissements contraires aux intérêts des populations du Tiers Monde : "Baby Killer" ou les méthodes publicitaires de Nestlé dans le Tiers Monde; des documents accablants montrant l'infiltration des firmes multinationales - suisses entre autres - dans I'organisation des Nations Unies; Brown Boveri au Brésil et le cartel mondial de l'électricité; Yamoussoukro ou I'hôpital de Côte d'Ivoire soutenu en Suisse dans le seul but de défendre quelques intérêts particuliers, etc...

- La participation à l'élaboration et au lancement d'une initiative populaire visant non pas à supprimer, comme le prétend la contre-propagande bancaire, mais à limiter le secret bancaire dont on sait qu'il attire et protège les revenus, souvent douteux, de certains personnages haut placés dans le Tiers Monde, peu soucieux de nuire ainsi à leur propre économie. La DB est aussi partie prenante de la campagne "retrait des fonds" visant à faire pression sur les trois grandes banques suisses qui ont des relations financières privilégiées avec l'Afrique du Sud.

- La mise sur pied d'un "symposium de la solidarité", avec d'autres organisations de développement, en Suisse romande, en 1978, puis au niveau national à Berne en mai 1981. "Le développement c'est la libération" proclame le Manifeste adopté en commun à cette occasion. 
- Dès le printemps 81 , lancement d'une large campagne "la faim est un scandale", visant à rendre plus explicites les raisons socio-économiques dans le monde. Premier exemple choisi, la production d'ananas aux Philippines par des firmes multinationales. Mobilisation des consommateurs (trices) suisses afin de faire pression sur les grandes firmes de distribution helvétiques pour qu'elles modifient leur politique d'achat dans ce domaine. Objectif à terme : I'introduction d'une "clause sociale" reconnaissant aux travailleurs (philippins en l'occurrence) un certain nombre de droits (salaires, emploi, achat de la terre...). L'utilisation sans discernement de pesticides dans le Tiers Monde par des firmes du Nord est également un volet important de cette campagne, appuyée non seulement dans les milieux tiers-mondistes, mais aussi par les milieux paysans, écologiques et des consommateurs.

- Parallèlement à des thèmes comme le secret bancaire, sujet brûlant s'il en est, des groupes analysent la littérature enfantine présentant le Tiers Monde aux enfants (une analyse critique est disponible sur demande en français et en allemand). Des jeux économiques sont réalisés afin de permettre aux groupes de comprendre rapidement les mécanismes du sousdéveloppement. Des montages audiovisuels et des dossiers pédagogiques sont diffusés. Avec d'autres organisations de coopération, la DB a également lancé un groupe "tourisme et développement" qui tente d'alerter les agences de voyage et leurs clients sur les problèmes posés par le tourisme dans le Tiers Monde. La DB a aussi participé à la création d'OS3, coopérative d'importation de produits du Tiers Monde. Enfin, elle s'associe aux objectifs et activités du Fonds de solidarité, créé en automne 1982, afin de soutenir la lutte pour la libération sociale dans le Tiers Monde, car sans libération "le combat contre la pauvreté se soldera par une défaite" (Manifeste du Symposium).

- Parce que racisme, xénophobie ou ethnocentrisme, où qu'ils s'expriment, sont condamnables, la DB est active dans le Communauté de travail "Etre solidaires", œuvrant pour une amélioration du statut des étrangers en Suisse.

- Par ses prises de position et ses informations, la DB cherche aussi à stimuler les partis politiques, les syndicats, les Eglises ou autres associations afin que le combat en Suisse pour le développement gagne de plus larges couches de la population. Elle met sur pied, à la demande, des soirées de formation et d'information sur ces problèmes dans les milieux les plus divers. 


\section{Une volonté de réflexion et de changement}

L'impasse du "développement" n'est plus contestée aujourd'hui. La situation de la majorité des populations du Tiers Monde ne fait que de se dégrader. Le fossé entre riches et pauvres s'accroît, à l'intérieur des pays comme entre le Nord et le Sud. Bien plus que d'une augmentation de l'aide, le développement exige surtout une volonté de réflexion et de changement.

Réflexion pour comprendre les causes et les effets du "maldéveloppement" du monde, changement aussi pour modifier les structures politico-économiques des pays industrialisés qui font obstacle à la libération des peuples. Les obstacles internes au Tiers Monde sont l'affaire des peuples du Tiers Monde; en revanche les obstacles externes souvent originaires du Nord - et dont l'influence négative sur les obstacles internes ne doit pas être sousestimée - sont notre affaire. La plus efficace coopération au développement, c'est de répondre aux nombreux appels venant du Tiers Monde nous demandant de changer d'abord chez nous les causes de leur misère :

"Exigez que notre travail soit correctement payé!

Mettez fin aux abus des multinationales et au pillage de nos matières premières.

Ne laissez pas donner asile à l'argent volé par nos dictateurs.

Ne laissez pas ceux qui chez vous sont responsables des décisions économiques soutenir ceux qui nous oppriment." 